Marquette University

e-Publications@Marquette

Biological Sciences Faculty Research and

Publications

Biological Sciences, Department of

$12-15-2018$

\title{
A Force Sensor that Converts Fluorescence Signal into Force Measurement Utilizing Short Looped DNA
}

Golam Mustafa

Kent State University

Cho-Ying Chuang

Michigan State University

William A. Roy

Kent State University

Mohamed M. Farhath

Kent State University

Nilisha Pokhrel

Marquette University

See next page for additional authors

Accepted version. Biosensors and Bioelectronics, Vol. 121 (December 15, 2018): 34-40. DOI. (C) 2018

Elsevier B.V. Used with permission. 


\section{Authors}

Golam Mustafa, Cho-Ying Chuang, William A. Roy, Mohamed M. Farhath, Nilisha Pokhrel, Yue Ma, Kazuo Nagawawa, Edwin Antony, Matthew J. Comstock, Soumitra Basu, and Hamza Balci 
Marquette University

\section{e-Publications@Marquette}

\section{Biology Faculty Research and Publications/College of Arts and Science}

This paper is NOT THE PUBLISHED VERSION; but the author's final, peer-reviewed manuscript.

The published version may be accessed by following the link in the citation below.

Biosensors and Bioelectronics, Vol. 121 (2018): 34-40. DOI. This article is (C) Elseiver and permission has been granted for this version to appear in e-Publications@Marquette. Elsevier does not grant permission for this article to be further copied/distributed or hosted elsewhere without the express permission from Elsevier.

\section{A force sensor that converts fluorescence signal into force measurement utilizing short looped DNA}

Golam Mustafa

Department of Physics, Kent State University, Kent, $\mathrm{OH}$

Cho-Ying Chuang

Department of Physics, Michigan State University, East Lansing, MI

William A. Roy

Department of Physics, Kent State University, Kent, $\mathrm{OH}$

Mohamed M. Farhath

Department of Chemistry and Biochemistry, Kent State University, Kent, $\mathrm{OH}$

Nilisha Pokhrel

Department of Biological Sciences, Marquette University, Milwaukee, WI

Yue Ma

Department of Biotechnology and Life Science, Tokyo University of Agriculture and Technology, Tokyo, Japan 


\section{Kazuo Nagasawa}

Department of Biotechnology and Life Science, Tokyo University of Agriculture and Technology, Tokyo, Japan

\section{Edwin Antony}

Department of Biological Sciences, Marquette University, Milwaukee, WI

\section{Matthew J. Comstock}

Department of Physics, Michigan State University, East Lansing, MI

\section{Soumitra Basu}

Department of Chemistry and Biochemistry, Kent State University, Kent, $\mathrm{OH}$

Hamza Balci

Department of Physics, Kent State University, Kent, $\mathrm{OH}$

\section{Abstract}

A force sensor concept is presented where fluorescence signal is converted into force information via single-molecule Förster resonance energy transfer (smFRET). The basic design of the sensor is a 100 base pair (bp) long double stranded DNA(dsDNA) that is restricted to a looped conformation by a nucleic acid secondary structure (NAS) that bridges its ends. The looped dsDNA generates a tension across the NAS and unfolds it when the tension is high enough. The FRET efficiency between donor and acceptor (D\&A) fluorophores placed across the NAS reports on its folding state. Three dsDNA constructs with different lengths were bridged by a DNA hairpin and $\mathrm{KCl}$ was titrated to change the applied force. After these proof-of-principle measurements, one of the dsDNA constructs was used to maintain the G-quadruplex (GQ) construct formed by thrombinbinding aptamer (TBA) under tension while it interacted with a destabilizing protein and stabilizing small molecule. The force required to unfold TBA-GQ was independently investigated with high-resolution optical tweezers (OT) measurements that established the relevant force to be a few $\mathrm{pN}$, which is consistent with the force generated by the looped dsDNA. The proposed method is particularly promising as it enables studying NAS, protein, and small molecule interactions using a highly-parallel FRET-based assay while the NAS is kept under an approximately constant force.

\section{Keywords}

Single molecule FRET; Force sensor; G-quadruplex; Optical tweezers; Looped dsDNA; Small molecule

\section{Introduction}

The polymer properties of long (length $>>$ persistence length, $I_{p}$ ) double stranded DNA (dsDNA) have been well described by the worm-like chain (WLC) model (Fixman and Kovac, 1973, Kovac and Crabb, 1982 ), and $I_{p} \approx 50 \mathrm{~nm} \approx 150$ base-pair (bp) in physiologically germane salt concentrations (Baumann et al., 1997, Bustamante et al., 1994). A short (length $\leq I_{p}$ ) dsDNA molecule is not expected to demonstrate significant bending due to thermal fluctuations. Nevertheless, bending of short dsDNA is frequently observed in physiological settings including wrapping of $146 \mathrm{bp}$ dsDNA around $10 \mathrm{~nm}$ size histones (Richmond and Davey, 2003) and packing of viral genome, $\sim 10 \mu \mathrm{m}$ in length, into a viral capsid of $\sim 50 \mathrm{~nm}$ radius (Chemla et al., 2005, Chemla and Smith, 2012). Transcription factors can bend dsDNA and form loops to bring different sites to close proximity as a way to regulate 
transcription (Kadauke and Blobel, 2009, Yadon et al., 2013). Significant bending of dsDNA has also been suggested to play a role in facilitating transition of proteins between two otherwise distal DNA binding sites (Jeong et al., 2016). However, these occurrences of dsDNA bending are facilitated by either the positively charged histones interacting with negatively charged DNA backbone, active packing of the viral genome into capsid by a motor protein, or activity of transcription factors. Nevertheless, they are also indicators for the possibility of restricting short dsDNA in a looped configuration in a properly designed assay.

Recent single molecule Förster Resonance Energy Transfer(smFRET) studies have succeeded in monitoring real time cyclization of isolated short dsDNA molecules (Vafabakhsh and $\mathrm{Ha}, 2012$ ). In these experiments, D\&A fluorophores are placed at the ends of a dsDNA molecule that has complementary 8$10 \mathrm{nt}$ overhangs, also called sticky-ends. When the dsDNA bends enough, the complementary stickyends meet and hybridize, giving rise to a loop structure and an abrupt increase in FRET efficiency (Le and Kim, 2014, Vafabakhsh and $\mathrm{Ha}, 2012$ ). Due to the significant bonding energy of complementary overhangs, the looped structure can be maintained for many seconds before it breaks and the process is repeated. By analyzing the time spent in high-FRET (looped) and low-FRET (linear) states, and the frequency of transitions between them, an estimate for the J-factor can be attained. J-factor is commonly interpreted as the effective concentration of one end of dsDNA in the vicinity of the other and is used as a reference for dsDNA bendability (Shore et al., 1981). These studies have suggested that short dsDNA has orders of magnitude higher bendability compared to what would be expected from WLC model (Vafabakhsh and Ha, 2012). In a similar assay, the looped state lifetime was used to calculate the shear force generated by dsDNA of a particular length (Jeong et al., 2016, Le and Kim, 2014). However, this extreme bendability has also been attributed to bp breaking due mismatches in synthetic DNA or thermal denaturation (Frank-Kamenetskii, 1997, Vologodskii and Frank-Kamenetskii, 2013, Wartell and Benight, 1985). The DNA looping assay was also used to analyze the impact of DNA modifications, such as methylation, on DNA flexibility and nucleosome stability (Ngo et al., 2016) and to study tension dependent enzyme kinetics (Joseph et al., 2014, Zocchi, 2009).

We present a new force sensor and transducer concept where the force generated by a looped short dsDNA is used to maintain a nucleic acid secondary structure (NAS) under an approximately constant tension, as averaged over measurement time ( $100 \mathrm{~ms}$ frame integration time). Fig. 1 illustrates this approach. The sequences of all constructs used in this study are given in Supplementary Materials. A short dsDNA with non-complementary sticky ends (SE1 and SE2) is hybridized to a bridging strand with end sequences that are complementary to SE1 and SE2. The center of this bridge strand contains a NAS, such as a hairpin, a G-quadruplex (GQ), or an RNA structure. The D\&A fluorophores are placed such that folded NAS results in higher FRET than unfolded NAS. In the looped state, the short dsDNA would maintain a tension across the ends of NAS and unfold it if the tension is large enough. Since it is elastically less demanding to maintain a longer dsDNA in a looped configuration, the force generated across the NAS is expected to decrease with dsDNA length. This force is also expected to decrease as the salt concentration is increased due to more efficient electrostatic shielding of the negative charges DNA backbone. However, varying the salt concentration might also influence the stability of NAS, convoluting the two effects. This issue will revisited in Section $\underline{4}$. Nevertheless, it should be clear that by placing an NAS across the ends of a short looped dsDNA, it is possible to maintain it under an approximately constant force. The proposed method addresses several noteworthy issues: (i) eliminates the need for additional instrumentation in order to generate a tension across an NAS by using a short looped dsDNA 
as a force transducer; (ii) significantly increases the throughputof force spectroscopy measurements; (iii) enables performing force measurements in low force regime, $\sim 1 \mathrm{pN}$, which is very challenging to achieve using other well-established methods.

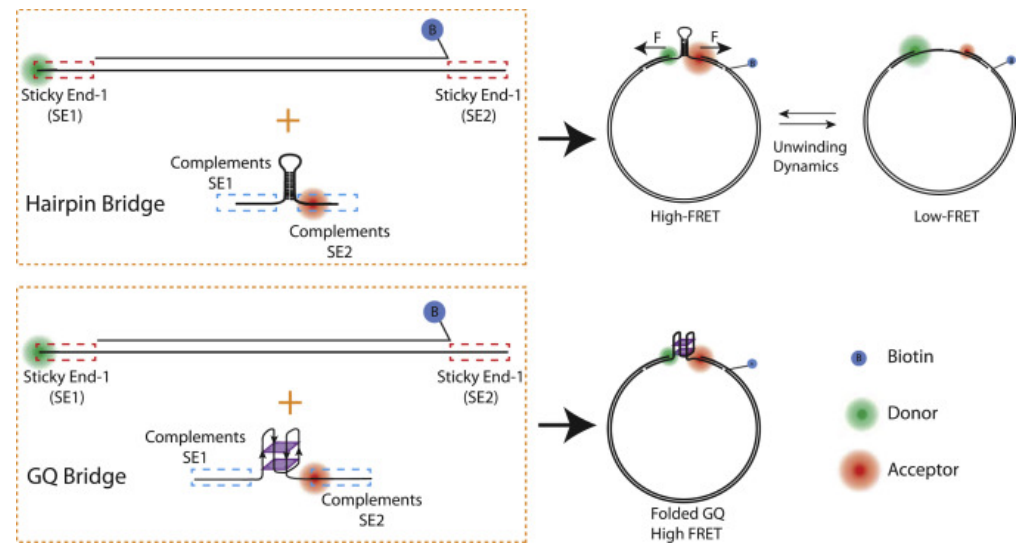

Fig. 1. A schematic illustration of how a short dsDNA and a bridge strand (top panel: hairpin, bottom panel: TBA$\mathrm{GQ}$ ) are combined via sticky ends to form a looped constructs.

\section{Material and methods}

Descriptions of the smFRET and OT assays are given in Supplementary Materials. The sequences of all DNA molecules and primers used for the PCR assays and a brief review of RPA purification protocol are also provided in Supplementary Materials.

The protocol to develop this method was optimized to attain maximum number of looped constructs however, it is possible for some constructs to bind a bridge strand but not form a loop and remain in the linear form. It is also possible for some constructs to bind two bridge strands, one on each end, which would also prevent loop formation. The NAS in such constructs might fold and show a high FRET state as it does not experience any tension in the non-looped (linear) state. These cases cannot be distinguished from those within a looped construct and therefore, need to be eliminated. In order to eliminate such constructs, we utilized an ssDNA that carries a fluorescencequencher and targets such non-looped constructs. The details of this assay and pictorial depiction of the need for a quencher are described in Supplementary Materials and Fig. S1.

\section{Results}

\subsection{Proof of principle measurements on a hairpin bridge}

In order to establish the proof of principle for the method, we utilized a 8-bp long hairpin with $50 \% \mathrm{GC}$ content as the bridge strand (8R50-4T construct in Woodside et al. (2006)). This length is similar to the length of complementary sticky ends used in earlier single molecule FRET studies even though the hairpin is unzipped in our construct while it is sheared in the other studies. Using the method described in Fig. 1, the hairpin bridge was connected across 70 bp, 90 bp, or 110 bp dsDNA molecules (Fig. 2).

These dsDNA molecules were amplified via polymerase chain reaction (PCR) from a pUC19 vector using the primers listed in Table S1. These constructs also have 15-nt SE1 and SE2 overhangs that hybridize with the ends of the bridge strand, adding another 30-bp to overall length. However, the endnucleotides of the bridge strand were not ligated to the dsDNA in order to maintain a uniform geometry and avoid a pitch mismatch at the intersection of the helices. Therefore, the constructs will be referred 
to with just the length of the dsDNA, excluding these 15-bp segments. Both fluorophores, Cy3 and Cy5, are placed at the end of these 15-bp regions, not within the primarily looped region (70 bp, $90 \mathrm{bp}$, or $110 \mathrm{bp}$ dsDNA), and are separated from the NAS within the bridge strand by two nucleotides. Therefore, even if the fluorophores are stacked on the short 15-bp duplexes, this should not influence the looped segment or the NAS. To prevent a bridge strand to bind to multiple dsDNA molecules and formation of long chains, the dsDNA are immobilized on the surface at a low density (many micrometers away from nearest neighbors) using biotin-neutravidin linker. The bridge strand is then introduced to the chamber, after excess unbounds dsDNA molecules are removed by a buffer exchange. A detailed description of this protocol is given in section A.4 of Supplementary Materials.
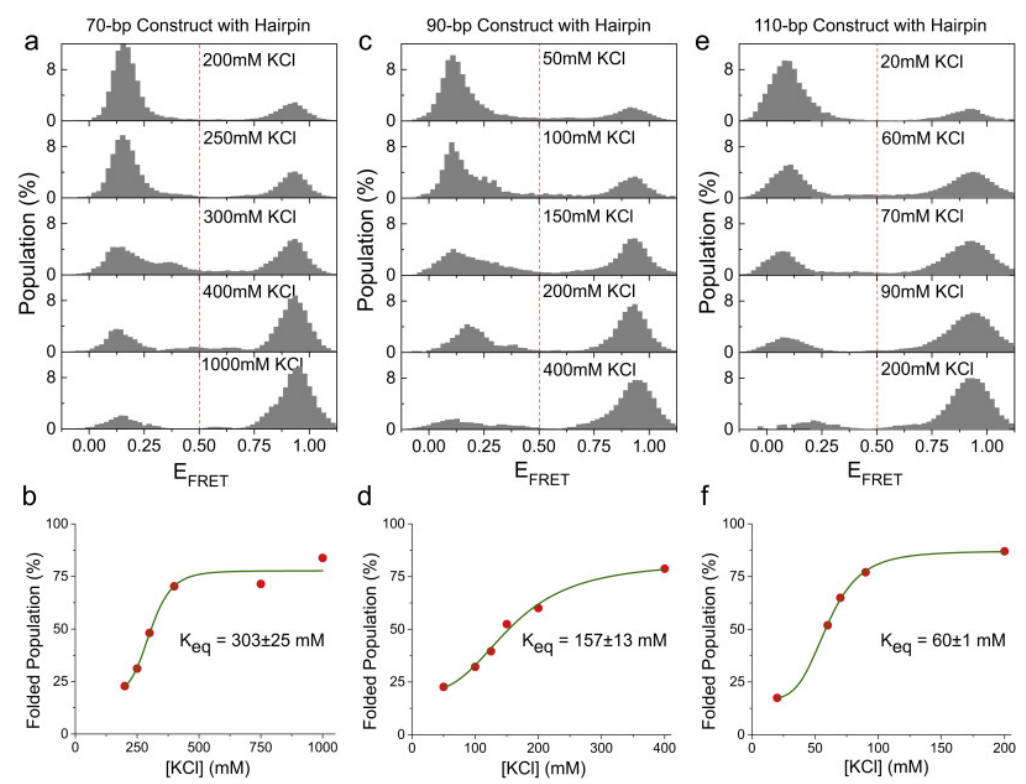

Fig. 2. Steady-state smFRET histograms for a 8-bp hairpin placed across a (a) 70-bp, (c) 90-bp, and (e) 110-bp long dsDNA. The hairpin transitions from the folded (high-FRET) to unfolded state (low-FRET) as the salt is reduced. A Hill equation fit to the folded state population as function of $[\mathrm{KCl}]$ results in $\mathrm{Keq}$ of $303 \mathrm{mM}, 157 \mathrm{mM}$, and $60 \mathrm{mM}$ $\mathrm{KCl}$ for 70-bp, 90-bp, and 110-bp dsDNA, respectively, as shown in (b), (d), and (f). The number of molecules in the histograms in Fig. 2a are 364, 372, 299, 186 and 75, sorted from low salt to high salt. Similarly, the number of molecules in the histograms in Fig. $2 \mathrm{c}$ are 230, 74, 107, 151, 160, and 103. For Fig. 2e, these numbers are: 92, 147, 148,144 , and 65.

We observe a transition from a high FRET state (folded) to a low FRET state (unfolded) as the salt concentration is reduced, as shown in Fig. 2a (70-bp construct), 2c (90-bp construct), and 2e (110-bp construct). Since the folded and unfolded states are well separated from each other on either side of $E_{F R E T}=0.50$, we determined the folded state population by integrating the population for $E_{F R E T} \geq 0.50$, and the unfolded state by integrating $\mathrm{E}_{\mathrm{FRET}}<0.50$ population. We plotted the folded state population as a function of $\mathrm{KCl}$ concentration $([\mathrm{KCl}])$ and fitted the resulting curve with Hill equation. These fits resulted in $\mathrm{K}_{\text {eq }}=303 \pm 25 \mathrm{mM}$ for 70-bp construct (Fig. 2b), $\mathrm{K}_{\text {eq }}=157 \pm 13 \mathrm{mM}$ for 90-bp construct (Fig. $2 \mathrm{~d}$ ), and $\mathrm{K}_{\mathrm{eq}}=60 \pm 1 \mathrm{mM}$ for 110-bp construct (Fig. 2e). The error bars are the standard deviation in the fitting parameters. A summary of fitting parameters is given in Supplementary Information Table S2.

Increasing the salt concentration results in lower applied forceon the NAS due to increased elasticity of dsDNA. However, it also results in a more stable hairpin as the repulsive interactionbetween the complementary segments of the hairpin are more effectively screened at higher salt. The salt 
dependence of unfolding force (Fu) was observed to be logarithmic (Huguet et al., 2010, Lee et al., 2006), resulting in small variation of about $1 \mathrm{pN}$ in $\mathrm{F}_{\cup}$ of this hairpin between $300 \mathrm{mM}$ and $60 \mathrm{mM} \mathrm{KCl}$, which is the relevant range for this study. A proper calibration of a particular dsDNA length requires deconvolution of the two effects, increased elasticity of dsDNA and higher stability of hairpin at higher salt. However, comparing the folded population at a particular $[\mathrm{KCl}]$, at which point the hairpin stability is constant across all three dsDNA constructs, would illustrate that the applied force is correlated with dsDNA length. To illustrate, at $200 \mathrm{mM} \mathrm{KCl}$, the folded population is $23 \%, 60 \%$, and $87 \%$ for 70-bp, 90-bp, and 110-bp dsDNA constructs, respectively. With these, the measurements on the hairpin construct demonstrate the following main premises of the method: (i) Looped dsDNA can be used to apply force on an NAS placed across its ends; (ii) The force generated by the looped dsDNA can be modulated by the salt concentration and dsDNA length; (iii) A FRET based approach can be used to attain force information in a highly parallel manner.

\subsection{Interactions of TBA-GQ with RPA and a small molecule}

Having established the proof-of-principle, we then utilized this method to study the unfolding characteristics of a GQ structure formed by thrombin binding aptamer (TBA-GQ). To the best of our knowledge, this challenging NAS had not been characterized with other force spectroscopy methods before. TBA-GQ is a two-tier GQ and is known to have a significantly lower thermal melting point ( $T_{m}=51^{\circ} \mathrm{C}$ at $100 \mathrm{mM} \mathrm{KCl}$ ) (Nagatoishi et al., 2011) compared to human telomeric GQ (hGQ), which forms a 3-tier $\mathrm{GQ}\left(\mathrm{T}_{\mathrm{m}}=68^{\circ} \mathrm{C}\right.$ at $100 \mathrm{mM} \mathrm{KCl}$ ) (Qureshi et al., 2012). hGQ is known to have $\mathrm{Fu}_{\mathrm{u}} \approx 20 \mathrm{pN}$ at physiological salt (Abraham Punnoose et al., 2014). Based on $T_{m}$ and tier number, we expect TBA-GQ to have a significantly smaller $F_{U}$ than $20 \mathrm{pN}$. Being able to detect nanometer scale structural changes at few $\mathrm{pN}$ force range would be fairly challenging for other force spectroscopy methods while it should be possible with this FRET-based approach (Budhathoki et al., 2016). To study TBA-GQ, we utilized the longest dsDNA construct (110-bp) that was characterized in Fig. 2 as it would enable accessing the lowest force regime around physiological salt. Fig. 3a shows steady state smFRET histograms at different $[\mathrm{KCl}]$ for TBA-GQ in the 110-bp construct. A clear transition from a folded to an unfolded state is observed as $[\mathrm{KCl}]$ is reduced. The folded state population was similarly determined by integrating the area for $E_{F R E T} \geq 0.50$. Fig. 3 b shows the corresponding Hill equation fit to folded population vs. [KCl], which results in $\mathrm{K}_{\mathrm{eq}}=75 \pm 6 \mathrm{mM}$. 

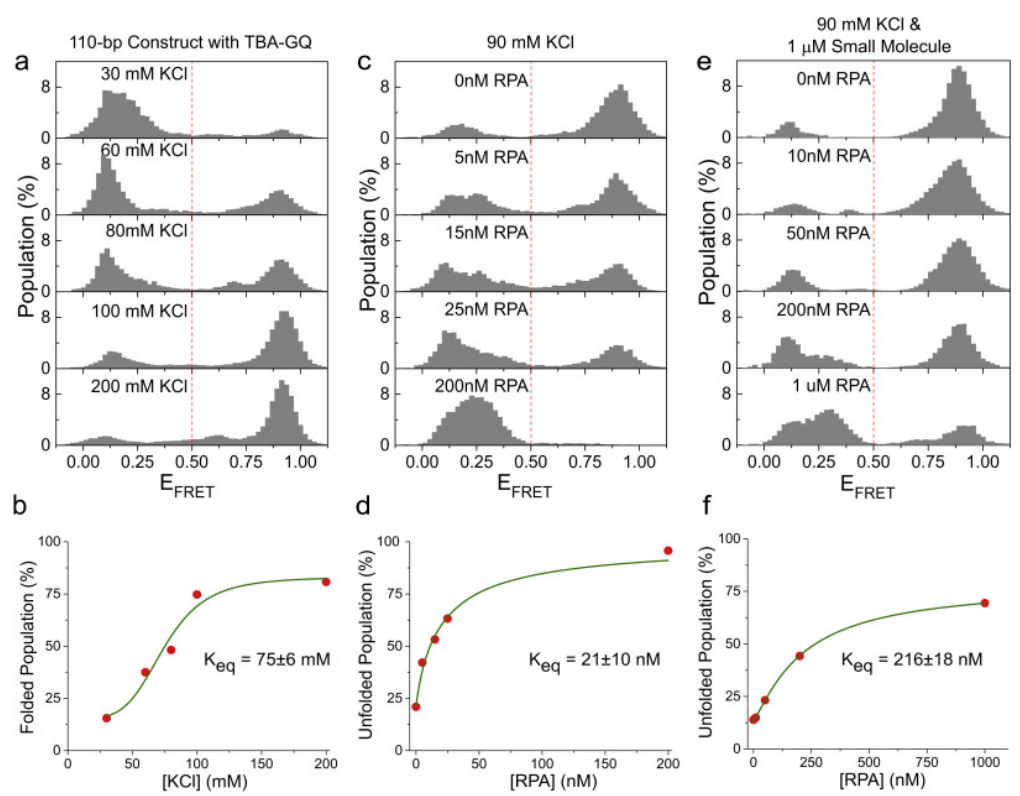

Fig. 3. Steady-state smFRET histograms for a TBA-GQ placed in a 110-bp dsDNA construct. (a) KCl titration, in the absence of any RPA and small molecule, shows a systematic transition from folded to unfolded state as the salt is reduced. The number of molecules in the histograms in Fig. 3 a are 95, 174, 151, 175, and 127, sorted from low salt to high salt. (b) A Hill equation fit to folded state population vs. [KCl] results in $\mathrm{Keq}_{\text {eq }}=75 \mathrm{mM}$. (c) RPA is titrated at $90 \mathrm{mM} \mathrm{KCl}$ in the absence of small molecule. Higher RPA concentration results in more TBA-GQ unfolding. The number of molecules in the histograms in Fig. $3 \mathrm{c}$ are 80, 97, 100, 63 and 76, sorted from low to high RPA concentration. (d) Hill equation fit to the unfolded population results in $K_{e q}=21 \mathrm{nM}$. (e) RPA is titrated in the presence small molecule L2H2-6OTD. The number of molecules in the histograms in Fig. 3 e are 90, 80, 95, 60 and 93, sorted from low to high RPA concentration. (f) Hill equation fit to the unfolded population results in $\mathrm{K}_{\text {eq }}=216 \mathrm{nM}$, which indicates an order of magnitude increase in stability of TBA-GQ in the presence of L2H2-6OTD.

We then tested the capabilities of the method to probe interactions of TBA-GQ with a small molecule and a protein while TBA-GQ is maintained under an approximately constant tension. Based on earlier studies, this tension could be estimated to be around a few pN (Le and Kim, 2014). A more precise estimate will be provided later via high-resolution OT measurements. Despite reliance of this FRET-based technique on OT-type methods in calibrating the force, such a calibration needs to be done only once. With such a calibration at hand, a given dsDNA construct could be used to maintain different NAS under that particular force and its interactions with proteins or small molecules could be studied using a highly-parallel FRET-based investigation. Higher or lower force values could be reached with shorter or longer dsDNA constructs, respectively.

Replication protein A (RPA) (Pokhrel et al., 2017, Sibenaller et al., 1998) is a ssDNA binding protein that is known to efficiently destabilize GQ structures (Ray et al., 2013). We used the 110-bp dsDNA and TBA$\mathrm{GQ}$ bridge for these measurements and titrated RPA at $90 \mathrm{mM} \mathrm{KCl}$, where most TBA-GQ molecules are folded before RPA is added ( $\underline{\text { Fig. } 3 \mathrm{C}}$ ). $90 \mathrm{mM} \mathrm{KCl}$ does not have any special significance and higher salt concentrations could have been used as well. As [RPA] is increased, the unfolded population is expected to increase while the folded population decreases. We plotted the unfolded state (total area under $E_{\text {FRET }}<0.5$ ) as a function of [RPA], and performed a Hill equation fit (Fig. $3 \mathrm{~d}$ ), which resulted in $\mathrm{K}_{\text {eq }}=21 \pm 10 \mathrm{nM}$. We then performed the same RPA titration at $90 \mathrm{mM} \mathrm{KCl}$ in the presence of $1 \mu \mathrm{M}$ L2H2-6OTD, a small molecule that stabilizes hGQ (lida and Nagasawa, 2013), but has not been tested 
before with TBA-GQ (Fig. 3e). Fig. $3 \mathrm{f}$ shows the unfolded population as a function of [RPA] and a Hill equation fit that resulted in $\mathrm{K}_{\mathrm{eq}}=216 \pm 18 \mathrm{nM}$. This $\mathrm{K}_{\mathrm{eq}}$ is an order of magnitude larger than that observed in the absence of L2H2-6OTD, in agreement with the small molecule stabilizing the TBA-GQ.

\subsection{High resolution optical tweezers measurements on TBA-GQ}

In order to put these measurements in better context and provide a measure of the forces generated by the looped DNA construct, we performed high-resolution optical tweezersmeasurements on TBA-GQ. A home-built timeshared dual-trap optical tweezers was used for these measurements (Comstock et al., 2011, Whitley et al., 2017), as briefly described in Supplementary Materials. The experimental design of the optical tweezers assay is shown in Fig. 4a inset. The TBA-GQ formed two G-tetrad layers at lower force and it unfolded at higher force. First, we performed non-equilibrium force ramp measurements at a constant speed of $100 \mathrm{~nm} / \mathrm{s}$ at $800 \mathrm{mM} \mathrm{KCl}$. Four representative tethers are shown in Fig. $4 \mathrm{a}$. The stepwise jumps between two polymer models represent the unfolding and refolding. The forceextension curves indicate that the TBA unfolded and refolded at a wide range of force, in agreement with observations with other GQ structures (Selvam et al., 2014). The folding/unfolding rates are slow compared to the scan speed. Therefore, the tethers showed heterogeneity in force-extension curves.
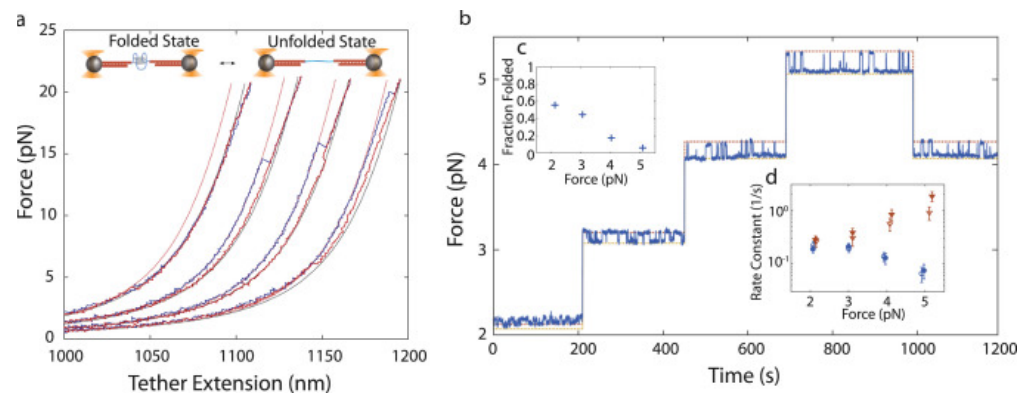

Fig. 4. (a) Non-equilibrium force ramp measurements of a single, representative TBA-GQ tether molecule. The inset cartoon shows the arrangement of the TBA-GQ tether molecule consisting of a single TBA-GQ attached to a pair of dsDNA handles and pulled on by a pair of trapped beads. Multiple pulling (blue) and relaxing (red) curves are shown with a $30 \mathrm{~nm}$ extension offset for clarity. The accompanying dashed lines are polymer models of the tether with the TBA-GQ folded (red, to the left) and unfolded (black, to the right). Pulling speed was $100 \mathrm{~nm} / \mathrm{s}$. (b-d) Equilibrium force-vs-time measurements of TBA-GQ tethers at $800 \mathrm{mM} \mathrm{KCl}$. b) Constant trap position measurements for a set of trap separations and corresponding mean forces $(2,3,4$ and $5 \mathrm{pN})$ for a single TBA-GQ molecule. The dashed lines are polymer models for folded and unfolded TBA-GQ (upper and lower lines respectively). Folded and unfolded dwell times change with force. c) Fraction-folded vs. force derived from 2state Gaussian fitting of histograms of raw data. d) Folding (blue circles) and unfolding (red triangles) rate constants vs. force derived from detailed dwell time analysis of folding and unfolding time trajectories (e.g., blue data trace). Filled markers are from an individual molecule and the open markers are the average for 6 unique molecules. Error bars are standard error of the mean.

To obtain equivalence force, we directly measured the time DNA spends in the folded and unfolded states under force in equilibrium by performing 'fixed trap position' measurements. This will be referred to as the 'dwell time' for particular state. Fig. 4b shows a typical TBA-GQ folded and unfolded time trajectoryin $800 \mathrm{mM} \mathrm{KCl}$ buffer. This higher salt concentration was used to facilitate identification of folding-unfolding events within a practical time interval. The expected force of TBA folding and unfolding was determined by calibrating its force-extension profiles. This long duration measurement demonstrates that the force changes of TBA folding and unfolding followed its polymer models and the TBA spent longer time at unfolded state when the force was increased. The fraction folded molecules 
was calculated from the histogram analysis (Fig. 4c). The histogram of force data was fitted to two Gaussian distributions, which represent folded state and unfolded state. The $50 \%$ folded force is about $2.5 \mathrm{pN}$ under $800 \mathrm{mM} \mathrm{KCl}$. Fig. $4 \mathrm{~d}$ shows the rate constants versus force. The rate constants of folding and unfolding are the inversion of dwell times. The folding and unfolding rate constant are force dependent. The dwell timeanalysis shows the transition between the equivalence of folded and unfolded states. The estimated equivalence force is also about $2.5 \mathrm{pN}$ that is consistent with the histogram analysis. The rate constants were consistent over many molecules. Similar experiments were performed at $3 \mathrm{pN}$ under a range of $\mathrm{KCl}$ concentration. This dwell time analysis (Fig. S2) shows that when $\mathrm{K}^{+}$ion concentration increases the folding rate constant increases, while the unfolding rate constant remains similar. The histogram analysis also suggests that the fraction folded increases when the $\mathrm{K}^{+}$ion concentration increases (Fig. S2b). As suggested by Fig. S2a, this increase is primarily due to increasing folding rate rather than decreasing unfolding rate.

Due to its very low stability, the folding-unfolding events of TBA-GQ were challenging to identify at lower $\mathrm{KCl}$ concentrations in the OT measurements. In smFRET measurements, the linear dsDNA constructs and bridge strands were incubated in $1 \mathrm{M} \mathrm{KCl}$ to enable loop formation, which also folds the TBA-GQ in the process, before lowering the salt concentration to the desired level. The system was then incubated at this lower salt concentration for about $30 \mathrm{~min}$ before surveying the folded and unfolded populations. Since such long time periods are not practical for OT measurements, higher salt concentration were used in Fig. 4. As folding and stability of $\mathrm{GQ}$ are primarily related to intercalation of

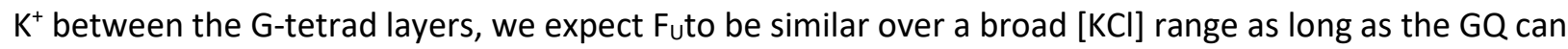
be successfully folded.

\section{Discussion}

The force generated by looped dsDNA has been estimated by measuring the time required to break a dsDNA loop held together by 10-bp long complementary sticky ends, using similar smFRET assays. The estimated force in these measurements was 3-4 pN for 100-bp long loops at $100 \mathrm{mM} \mathrm{NaCl}$. The results of shear force calculations using Monte-Carlo simulations assuming WLC or kWLC models for this loop lengthand salt concentration were also similar (Le and Kim, 2014). However, this force is based on joining the complementary sticky ends of the dsDNA, i.e. end to end separation $\approx 0$. In our case, the sticky ends of the dsDNA are not complementary and are bridged with a NAS and 30-bp dsDNA, which requires less bending of the structure and results in a lower force. Based on these arguments, the force generated by our 110-bp dsDNA construct should be less than $3 \mathrm{pN}$ at physiological salt. The OT measurements demonstrate the unfolding force for TBA-GQ to be $2.5 \mathrm{pN}$ at $800 \mathrm{mM} \mathrm{KCl}$ (Fig. 4c). In addition, Fig. S2a shows that the unfolding rate constant does not show significant variation over 150$900 \mathrm{mM} \mathrm{KCl}$. The similarity of the unfolding rate constant suggests that the unfolding force would be $\approx 2.5 \mathrm{pN}$ over $150-900 \mathrm{mM} \mathrm{KCl}$ as well. Fig. 3a shows that the majority of TBA-GQ molecules are folded at $150 \mathrm{mM} \mathrm{KCl}$, suggesting the force generated by the 110-bp construct is less than $2.5 \mathrm{pN}$ at this [KCl]. However, the folded and unfolded state populations are equal at $75 \mathrm{mM} \mathrm{KCl}$ ( $\underline{\mathrm{Fig}}$. $3 \mathrm{~b}$ ), suggesting the force generated by the $110-\mathrm{bp}$ dsDNA to be approximately $2.5 \mathrm{pN}$ at this salt.

A related issue in this respect is the variation in the folded TBA-GQ fraction in the OT and looped dsDNA measurements. As shown in Fig. S2b, most molecules are unfolded at $150 \mathrm{mM} \mathrm{KCl}$ when a constant $3 \mathrm{pN}$ force is applied in OT measurements. However, Fig. 3a shows that most molecules are folded in the looped dsDNA construct even at $100 \mathrm{mM} \mathrm{KCl}$. One aspect of this was already explained above as the 
looped dsDNA applies $2.5 \mathrm{pN}$ or less at $100 \mathrm{mM} \mathrm{KCl}$. The other aspect is related to how the force is applied in OT vs. looped dsDNA assays. In OT measurements, a constant force of $3 \mathrm{pN}$ is applied regardless of whether the TBA-GQ is folded or unfolded. However, in the looped dsDNA assays, the force that is applied after TBA-GQ is unfolded is significantly less than that in the folded state. Unfolding of the TBA-GQ increases the end-to-end separation of the looped dsDNA and requires less bending of it, which results in a significantly lower force. Under these conditions, it is easier for the TBA-GQ to refold in the looped dsDNA assay compared to the OT assay.

The calibration process with the OT has been challenging due to the low magnitude of forces that are required to unfold the TBA-GQ. However, the capability to maintain an NAS under a low-magnitude (on the order of $1 \mathrm{pN}$ ), constant tension might be adequate in many applications and knowing the exact magnitude of tension may not be crucial. Such studies would typically be comparative, where relative impact of different agents is investigated. For example, identifying the most prominent small molecule, which stabilizes a particular NAS most effectively, may not require an exact knowledge of the applied force on the NAS. As long as the candidate molecules are compared under identical conditions, it should be possible to compare their relative impact. Similarly, investigating whether two proteins positively or negatively cooperate to destabilize or bind to an NAS that is kept under tension may not require an exact knowledge of the applied force. In a recent study, a similar approach was used to identify certain DNA modifications that made dsDNA stiffer while other made it softer by monitoring the change in the looping probability (Ngo et al., 2016). As illustrated by these examples, the complications associated with the calibration process may not necessarily prohibit utilizing this method for different applications.

The capability to control the end-to-end separation of the looped dsDNA in our construct could be utilized to compare the force generated by looped dsDNA at specific end-to-end distances, which is of fundamental significance. This could be done by introducing additional nucleotides between the sticky ends (SE1 and SE2) and the NAS. In our current design, we have two unpaired nt on either side of the NAS. However, more nucleotides could be introduced to increase the end-to-end separation of the dsDNA, and generate smaller magnitudes of force. The change in the applied force can be quantified based on fraction of folded NAS molecules. As the optimal length of dsDNA for different applications is not universal and needs to be adjusted based on the desired force, this provides another knob, in addition to dsDNA length and salt concentration, to achieve this control.

It is known that a higher salt concentration would not only result in a more flexible looped dsDNA but also more stable hairpin construct. Therefore, the two effects complement each other and both result in more folded population at higher salt, which complicates the calibration process. In such cases varying the force by varying the length of the looped dsDNA, while maintaining a fixed salt concentration, could be used as a remedy. Another practical alternative to this approach is to use the same looped dsDNA length but add additional unpaired ssDNA spacers to the bridge strand. This will increase the end-to-end separation required for looped configuration and should result in less force on the NAS. In the case of GQs, the force can also be modulated by using the same concentration of different ions, e.g. $\mathrm{K}^{+}, \mathrm{Na}^{+}$, or $\mathrm{Li}^{+}$. Having said these, there are many applications in which the method could be used at a fixed salt concentration while varying the concentration of a protein or small molecule that primarily target the NAS. This approach enables performing NAS-protein or small molecule interactions under an approximately constant force, which are otherwise very challenging to perform. 
Despite the long incubation times before data acquisition, we occasionally observed dynamic transitions between different FRET levels for both the hairpin and the TBA-GQ bridge within our imaging time of 2$3 \mathrm{~min}$ (Supplementary Materials Fig. S3). The number of such dynamic traces was not adequate to perform a detailed analysis. We also tested the limiting case of a very stable $G Q$ that is expected to remain folded over a broad range of ionic conditions. We used the $G Q$ formed by sequence GGGTGGGTGGGTGGG (called 3Ly1Lp in Supplementary Materials Fig. S4), which is known to have a thermal melting temperature of $\mathrm{T}_{\mathrm{m}}=84^{\circ} \mathrm{C}$ even at $5 \mathrm{mM} \mathrm{KCl}$, and $\mathrm{T}_{\mathrm{m}}>94 \mathrm{C}$ at $15 \mathrm{mM} \mathrm{KCl}$ (Mathias et al., 2014). To test the extreme conditions, we performed measurements in $\mathrm{LiCl}$ rather than $\mathrm{KCl}$ since $\mathrm{Li}^{+}$is a significantly weaker stabilizer of GQ compared to $\mathrm{K}^{+}$(Maleki et al., 2017). Supplementary Materials Fig. $\underline{\mathrm{S}}$ shows that $3 \mathrm{Ly} 1 \mathrm{Lp}$ construct remains folded even at $2 \mathrm{mM} \mathrm{LiCl}$, confirming the expectations.

Presence of a surface in the immediate vicinity of the looped dsDNA would influence the conformational space of dsDNA and might give rise to variations in the force applied by the looped dsDNA (Moiseev et al., 2006, Tsortos et al., 2008). To minimize the effect of the surface, we have used PEG surface passivationand have attached the looped dsDNA to PEG via biotin-neutravidin linker. The biotin linker that is attached to the dsDNA has a long, 15-atom, spacer (biotin-TEG from IDT-DNA). Therefore, even though the impact of the surface cannot be completely eliminated, we believe the PEG coating and the long biotin spacer would reduce these interactions to below the levels commonly encountered in single molecule assays.

Some histograms (Figs. 2a or $\underline{3}$ C) and single molecule traces (Fig. S3a) show intermediate FRET states that are not expected based on a simple two state folded/unfolded model. The cause of these intermediate states is not clear at this point as partially unfolded states are not expected for such a short hairpin or a two-layer GQ. Photophysical effects due to fluorophore-DNA interactions might be a potential cause. However, more work in terms of designing different constructs with different linkers would be needed to identify the source of these intermediate FRET states. However, it should be clear that these are minor populations which do not influence the primary aim of this manuscript in establishing the applicability of proposed method.

\section{Conclusions}

Measuring the force via a fluorescent signal provides significant advantages. The parallel nature of smFRET measurements greatly accelerates the data acquisition process. With the described protocol, we monitored 20-60 molecules simultaneously, and reported force information obtained from several hundred single molecules for each condition, which is an order of magnitude larger than what is typically reported in other techniques. Even though a reasonable measure of the force for looped dsDNA is currently available for only a limited rangeof loop lengths and ionic conditions, these gaps could be filled with more comparative measurements using this method and other force spectroscopy methods. Once characterized, a given dsDNA loop can be used to study various NAS without the need for recalibration. The accessibility of the required instrumentation makes this method a promising complement to highresolution force spectroscopy methods, especially in the low force regime $(F<10 \mathrm{pN})$ which is more challenging for other methods. Hairpins, GQs, RNA secondary structures, or Holliday junctions are some of the potential NAS that can be studied with this approach. Finally, being able to measure the force generated by a looped dsDNA of a particular length opens up possibilities for modeling efforts that aim to understand fundamental polymer properties of short dsDNA, which remains to be an active research field. 


\section{Acknowledgements}

This work was supported by National Institutes of Health, USA [1R15GM109386 and 1R15GM123443 to H.B. and 7R15GM110671to E.A.], National Science Foundation, USA [NSF MCB-1514706 to M.J.C.], and Kent State University Research Council [to H.B. and S.B.]. Funding for open access charge: National Institutes of Health.

\section{Conflict of interest}

The authors declare no competing financial interest.

\section{Appendix A. Supplementary material}

https://ars.els-cdn.com/content/image/1-s2.0-S0956566318306821-mmc1.pdf

\section{References}

Abraham Punnoose et al., 2014J. Abraham

Punnoose, Y. Cui, D. Koirala, P.M. Yangyuoru, C. Ghimire, P. Shrestha, H. Mao. Interaction of Gquadruplexes in the full-length $\mathbf{3}^{\prime}$ human telomeric overhang. J. Am. Chem.

Soc., 136 (52) (2014), pp. 18062-18069

Baumann et al., 1997 C.G. Baumann, S.B. Smith, V.A. Bloomfield, C.Bustamante. Ionic effects on the elasticity of single DNA molecules. Proc. Natl. Acad. Sci. USA, 94 (12) (1997), pp. 6185-6190

Budhathoki et al., 2016 J.B. Budhathoki, P. Maleki, W.A. Roy, P. Janscak, J.G. Yodh, H. Balci. A

Comparative study of G-quadruplex unfolding and DNA reeling activities of human RECQ5 helicase. Biophys. J., 110 (12) (2016), pp. 2585-2596

Bustamante et al., 1994 C. Bustamante, J.F. Marko, E.D. Siggia, S. Smith. Entropic elasticity of lambdaphage DNA. Science, 265 (5178) (1994), pp. 1599-1600

Chemla et al., 2005

Y.R. Chemla, K. Aathavan, J. Michaelis, S. Grimes, P.J.Jardine, D.L. Anderson, C. Bustamante. Mechanism of force generation of a viral DNA packaging motor. Cell, 122 (5) (2005), pp. 683692

Chemla and Smith, 2012Y.R. Chemla, D.E. Smith. Single-molecule studies of viral DNA packaging. Adv. Exp. Med. Biol., 726 (2012), pp. 549-584

Comstock et al., 2011 M.J. Comstock, T. Ha, Y.R. Chemla. Ultrahigh-resolution optical trap with singlefluorophore sensitivity. Nat. Methods, 8 (4) (2011), pp. 335-340

Fixman and Kovac, 1973 M. Fixman, J. Kovac. Polymer conformational statistics. III. Modified Gaussian models of stiff chains. J. Chem. Phys., 58 (4) (1973), pp. 1564-1568

Frank-Kamenetskii, 1997 M.D. Frank-Kamenetskii. I.M. Lifshitz and condensed matter TheoryBiophysics of the DNA molecule. Phys. Rep., 288 (1) (1997), pp. 13-60

Huguet et al., 2010 J.M. Huguet, C.V. Bizarro, N. Forns, S.B. Smith, C.Bustamante, F. Ritort. Singlemolecule derivation of salt dependent base-pair free energies in DNA. Proc. Natl. Acad. Sci. USA, 107 (35) (2010), pp. 15431-15436

lida and Nagasawa, 2013 K. lida, K. Nagasawa. Macrocyclic polyoxazoles as G-quadruplex ligands. Chem. Rec., 13 (6) (2013), pp. 539-548

Jeong et al., 2016 J. Jeong, T.T. Le, H.D. Kim. Single-molecule fluorescence studies on DNA looping. Methods, 105 (2016), pp. 34-43 
Joseph et al., 2014 C. Joseph, C.Y. Tseng, G. Zocchi, T. Tlusty. Asymmetric effect of mechanical stress on the forward and reverse reaction catalyzed by an enzyme. PLoS One, 9 (7) (2014), p. e101442

Kadauke and Blobel, 2009 S. Kadauke, G.A. Blobel Chromatin loops in gene regulation. Biochim.

Biophys. Acta, 1789 (1) (2009), pp. 17-25

Kovac and Crabb, 1982 J. Kovac, C.C. Crabb. Modified Gaussian model for rubber elasticity. 2. The wormlike chain. Macromolecules, 15 (2) (1982), pp. 537-541

Le and Kim, 2014 T.T. Le, H.D. Kim Probing the elastic limit of DNA bending. Nucleic Acids Res., 42 (16) (2014), pp. 10786-10794

Lee et al., 2006 C.H. Lee, C. Danilowicz, R.S. Conroy, V.W. Coljee, M.Prentiss. Impacts of magnesium ions on the unzipping of $\boldsymbol{\lambda}$-phage DNA. J. Phys.: Condens. Matter, 18 (14) (2006), p. S205

Maleki et al., 2017 P. Maleki, J.B. Budhathoki, W.A. Roy, H. Balc. iA practical guide to studying Gquadruplex structures using single-molecule FRET. Mol. Genet. Genom., 292 (3) (2017), pp. $483-498$

Mathias et al., 2014 J. Mathias, R. Okyere, L. Lomidze, D. Gvarjaladze, K.Musier-Forsyth, B. Kankia. Thermal stability of quadruplex primers for highly versatile isothermal DNA amplification. Biophys. Chem., 185 (2014), pp. 14-18

Moiseev et al., 2006 L. Moiseev, M.S. Unlu, A.K. Swan, B.B. Goldberg, C.R. Cantor. DNA conformation on surfaces measured by fluorescence self-interference. Proc. Natl. Acad. Sci. USA, 103 (8) (2006), pp. 2623-2628

Nagatoishi et al., 2011 S. Nagatoishi, N. Isono, K. Tsumoto, N. Sugimoto. Loop residues of thrombinbinding DNA aptamer impact G-quadruplex stability and thrombin binding. Biochimie, 93 (8) (2011), pp. 1231-1238

Ngo et al., 2016 T.T. Ngo, J. Yoo, Q. Dai, Q. Zhang, C. He, A. Aksimentiev, T. Ha. Effects of cytosine modifications on DNA flexibility and nucleosome mechanical stability. Nat. Commun., 7 (2016), p. 10813

\section{Pokhrel et al., 2017}

N. Pokhrel, S. Origanti, E.P. Davenport, D. Gandhi, K.Kaniecki, R.A. Mehl, E.C. Greene, C. Dockend orff, E. Antony. Monitoring Replication Protein A (RPA) dynamics in homologous recombination through site-specific incorporation of non-canonical amino acids. Nucleic Acids Res., 45 (16) (2017), pp. 9413-9426

Qureshi et al., 2012M.H. Qureshi, S. Ray, A.L. Sewell, S. Basu, H. Balci. Replication protein A unfolds Gquadruplex structures with varying degrees of efficiency. J. Phys. Chem. B, 116 (19) (2012), pp. 5588-5594

Ray et al., 2013 S. Ray, M.H. Qureshi, D.W. Malcolm, J.B. Budhathoki, U.Celik, H. Balci. RPA-mediated unfolding of systematically varying G-quadruplex structures. Biophys. J., 104 (10) (2013), pp. 2235-2245

Richmond and Davey, 2003 T.J. Richmond, C.A. Davey. The structure of DNA in the nucleosome core. Nature, 423 (6936) (2003), pp. 145-150

Selvam et al., 2014 S. Selvam, D. Koirala, Z. Yu, H. Mao. Quantification of topological coupling between DNA superhelicity and G-quadruplex formation. J. Am. Chem. Soc., 136 (40) (2014), pp. 1396713970

Shore et al., 1981 D. Shore, J. Langowski, R.L. Baldwin. DNA flexibility studied by covalent closure of short fragments into circles. Proc. Natl. Acad. Sci. USA, 78 (8) (1981), pp. 4833-4837 
Sibenaller et al., 1998 Z.A. Sibenaller, B.R. Sorensen, M.S. Wold. The 32- and 14-kilodalton subunits of replication protein $A$ are responsible for species-specific interactions with single-stranded DNA. Biochemistry, 37 (36) (1998), pp. 12496-12506

Tsortos et al., 2008 A. Tsortos, G. Papadakis, K. Mitsakakis, K.A. Melzak, E. Gizeli. Quantitative determination of size and shape of surface-bound DNA using an acoustic wave sensor. Biophys. J., 94 (7) (2008), pp. 2706-2715

Vafabakhsh and $\mathrm{Ha}, 2012$ R. Vafabakhsh, T. Ha. Extreme bendability of DNA less than 100 base pairs

long revealed by single-molecule cyclization. Science, 337 (6098) (2012), pp. 1097-1101

Vologodskii and Frank-Kamenetskii, 2013 A. Vologodskii, M.D. Frank-Kamenetskii. Strong bending of the DNA double helix. Nucleic Acids Res., 41 (14) (2013), pp. 6785-6792

Wartell and Benight, 1985 R.M. Wartell, A.S. Benight. Thermal denaturation of DNA molecules: a comparison of theory with experiment. Phys. Rep., 126 (2) (1985), pp. 67-107

Whitley et al., 2017 K.D. Whitley, M.J. Comstock, Y.R. Chemla. High-resolution optical tweezers combined with single-molecule confocal microscopy. Methods Enzymol., 582 (2017), pp. 137169

Woodside et al., 2006 M.T. Woodside, W.M. Behnke-

Parks, K. Larizadeh, K. Travers, D. Herschlag, S.M. Block Nanomechanical measurements of the sequence-dependent folding landscapes of single nucleic acid hairpins. Proc. Natl. Acad. Sci. USA, 103 (16) (2006), pp. 6190-6195

Yadon et al., 2013 A.N. Yadon, B.N. Singh, M. Hampsey, T. Tsukiyama. DNA looping facilitates targeting of a chromatin remodeling enzyme. Mol. Cell, 50 (1) (2013), pp. 93-103

Zocchi, 2009 G. Zocchi. Controlling proteins through molecular springs. Annu. Rev. Biophys., 38 (2009), pp. $75-88$ 